\title{
Mudanças climáticas na educação: um levantamentodas práticas, ferramentas e tecnologias digitais
}

\author{
Katyeudo Karlos de S. Oliveira ${ }^{1}$, Ricardo André C. de Souza ${ }^{1,2}$ \\ ${ }^{1}$ Programa de Pós-Graduação em Informática Aplicada (PPGIA) \\ ${ }^{2}$ Departamento de Computação (DC) \\ Universidade Federal Rural de Pernambuco(UFRPE) \\ karlos.1914.so@gmail.com, ricardo.souzadufrpe.br
}

\begin{abstract}
The consequences of climate change affect everything from people's well-being to the businesses sustainability. Education is a means to mitigate the anthropic actions that cause climate change, whether by environmental awareness or by understanding the impacts of climate factors on social, chemical, biological, geographic processes, etc. There are some educational approaches, such as Environmental Education, Climate Change Education and Education for Sustainable Development that are concerned with addressing climate change in the teaching and learning process, but do not specify the practical tools that can be used by educators. This work then presents a survey of the practices, tools and digital technologies used in the educational environment to address the causes and effects of climate change, resulting from a systematic literature mapping.
\end{abstract}

Resumo. As consequências das mudanças climáticas afetam desde o bemestar das pessoas até a sustentabilidade dos negócios. A educação é um meio para mitigar as ações antrópicas que causam as mudanças climáticas, seja pela conscientização ambiental ou pela compreensão dos impactos dos fatores climáticos nos processos sociais, químicos, biológicos, geográficos, etc. Existem algumas abordagens educacionais tais como, Educação Ambiental (EA), Climate Change Education (CCE) e Educação para o Desenvolvimento Sustentável (EDS), que se preocupam em tratar das mudanças climáticas no processo de ensino e aprendizagem, porém não especificam os meios práticos que podem ser usados pelos educadores. Este trabalho apresenta então um levantamento das práticas, ferramentas e tecnologias digitais usadas no ambiente educacional para tratar das causas e efeitos das mudanças climáticas, resultante de um mapeamento sistemático da literatura.

\section{Introdução}

As mudanças climáticas são temas centrais dos debates globais. Educadores, cientistas e formuladores de políticas ainda possuem uma percepção limitada do que deve ser discutido sobre as mudanças climáticas em associação à educação [Mochizuki e Bryan 2015]. Diversos métodos estão sendo utilizados no combate às mudanças climáticas [IPCC 2019; ONU 2020]. A educação é vista como um dos métodos que podem auxiliar no processo de aprendizado sobre as causas e ameaças das mudanças climáticas [Cherry 2011; Sharma 2012]. As mudanças climáticas estão associadas aos fatores de tempo e clima. O tempo é o estado momentâneo da atmosfera, já o clima, é o estado médio da 
atmosfera que é medido por intermédio dos eventos temporais por um longo período [Reboita et al. 2012]. Conforme o IPCC (2007), mudança climática é uma variação em longo prazo estatisticamente significante em um parâmetro climático (como temperatura, precipitação ou ventos) médio ou na sua variabilidade, durante um período de tempo extenso. A mudança climática pode ser causada por processos naturais da própria Terra ou por forças externas, incluindo variações na intensidade da luz solar, ou ainda pela ação antrópica (causada pelo homem). Entre as consequências mais conhecidas das mudanças climáticas estão o aquecimento global, a desertificação, e a elevação da temperatura da água do mar.

Tomar medidas urgentes para combater a mudança climática e seus impactos é um dos Objetivos de Desenvolvimento Sustentável (ODS 13) da Agenda 2030 da Organização das Nações Unidas (ONU) [Pinho e Granziera 2019]. Para alcançar este objetivo, uma das metas consiste em melhorar a educação, elevar a conscientização e o conhecimento das pessoas e instituições sobre mitigação, adaptação, redução dos efeitos e alerta prévio das mudanças climáticas [ONU 2020].

A mudança climática é um conteúdo complexo, com ligações socioecológicas relevantes. Competências para resolução de problemas e tomada de decisão são vita is em vários níveis, desde a comunidade científica até o cidadão. Para decidir sobre questões climáticas, é necessário conhecer as causas que as motivam e a contribuição das ações diárias que afetam o problema. Avaliações das causas e efeitos das alterações no clima necessitam de abordagens inovadoras [Visintainer e Linn 2015]. À medida que as mudanças climáticas tornam-se cada vez mais presentes no dia-a-dia e demandam por soluções inovadoras também na área de educação, ferramentas e abordagens pedagógicas mais eficazes envolvendo o processo de ensino-aprendizagem estão s en do criadas e estudadas [Puttick e Tucker-Raymond 2018]. Os novos conhecimentos e competências exigirão um tratamento educacional plurifacetado direcionado para pessoas de qualquer idade, com respostas mais explícitas, incluindo alunos de todos os níveis do sistema educacional [Mochizuki e Bryan 2015].

Diante deste contexto, neste trabalho são expostos a sistemática e os resultados de um mapeamento da literatura para identificação das práticas, ferramentas e tecnologias digitais usadas no ambiente educacional usadas para melhorar a conscientização e compreensão das causas e consequências das mudanças climáticas. $\mathrm{O}$ artigo é organizado em cinco seções, a começar desta introdução. A Seção 2 retrata os pontos principais do protocolo de pesquisa e como o mapeamento foi ex ecutado. Em seguida, na Seção 3, os resultados do estudo são expostos. Na Seção 4, é realizada a discussão dos resultados. Por fim, na Seção 5 são feitas as considerações finais.

\section{Mapeamento Sistemático da Literatura}

O mapeamento conduzido foi elaborado com base no método proposto por Peters en et al. (2008), ao passo que, há quatro estágios fundamentais a serem seguidos: (i) definição do escopo da pesquisa, (ii) busca de estudos primários relevantes, (iii) extração de dados, e (iv) análise e síntese dos estudos. 


\subsection{Definição do Escopo do Mapeamento}

Neste estágio, foi executado o planejamento do mapeamento sistemático, por meio de um protocolo (Tabela 1) contendo a estratégia para a busca e uma questão de pesquisa (Research Question - RQ) com o objetivo de guiar o processo.

Tabela 1: Protocolo para o Mapeamento Sistemático da Literatura

\begin{tabular}{|c|c|}
\hline \multicolumn{2}{|c|}{$\begin{array}{l}\text { Questão de Pesquisa: Quais as práticas, ferramentas e tecnologias digitais usados nas } \\
\text { abordagens educacionais voltadas para conscientização e entendimento das mudanças } \\
\text { climáticas? }\end{array}$} \\
\hline \multicolumn{2}{|c|}{ Estratégia de Busca } \\
\hline Bases de Dados Científicas & Equipe Executora \\
\hline $\begin{array}{l}\text { IEEE Xplore Digital Library; Springer Link; } \\
\text { ACM Digital Library; Scopus; Scielo; SAGE } \\
\text { journals; Holos. }\end{array}$ & $\begin{array}{l}\text { 1. Estudante de pós-graduação; } \\
\text { 2. Professor orientador. }\end{array}$ \\
\hline Anos de Publicação & 2014 a 2019 \\
\hline Keywords & Palavras-chave \\
\hline $\begin{array}{l}\text { "action learning", "action-based learning", } \\
\text { "active learning", "climate change } \\
\text { education", "empathy-based learning", } \\
\text { "environmental education", "reflection } \\
\text { learning" }\end{array}$ & $\begin{array}{l}\text { "aprendizado de ação", "aprendizado de reflexão", } \\
\text { "aprendizagem ativa", "aprendizagem baseada em } \\
\text { ação", "aprendizagem baseada em em patia", } \\
\text { "educação ambiental", "educação sobre mudanças } \\
\text { climáticas" }\end{array}$ \\
\hline \multicolumn{2}{|c|}{ Método de Execução da Busca } \\
\hline \multicolumn{2}{|c|}{$\begin{array}{l}\text { 1) Pesquisar nas bases de dados através da string de busca; } \\
\text { 2) Analisar os títulos e resumos dos estudos primários retornados nas buscas e selecionar } \\
\text { aqueles que atendem os critérios de exclusão; } \\
\text { 3) Analisar o corpo do texto dos estudos selecionados e aplicar os critérios de qualidade para } \\
\text { identificar os aceitos e rejeitados no contexto do mapeamento sistemático da literatura. }\end{array}$} \\
\hline
\end{tabular}

\subsection{Busca por Estudos Primários}

Neste estágio, foi executada a busca nas bases de pesquisa para obter os estudos. Os resultados da busca visando responder a RQ são caracterizados como Estudos Primários (EP) [Magalhães et al. 2013].O desenvolvimento da string consistiu na utilização das palavras-chave nos idiomas português e inglês, segundo a RQ, com os conectores lógicos OR e AND (Tabela 2). Após a execução da string, retornaram 192 EP.

Tabela 2: String de Busca da Pesquisa

\begin{tabular}{|c|c|}
\hline ("climate change education" OR & ("educação sobre mudanças climáticas" OR \\
"environmental education") AND ("action & "educação ambiental") AND ("aprendizado de \\
learning" OR "action-based learning" OR & ação" OR "aprendizagem baseada em ação" OR \\
"active learning" OR "empathy-based & "aprendizagem ativa" OR "aprendizagem baseada \\
learning" OR "reflection learning") & em empatia" OR "aprendizado de reflexão") \\
\hline
\end{tabular}

\subsection{Extração de Dados}

Neste estágio, buscou-se adquirir informações dos EP encontrados, utilizando os critérios de exclusão: EP escrito em outra língua que não a inglesa e portuguesa; EP não relacionado ao tema da pesquisa; EP repetido; e EP que não seja artigo completo, tais como: resumo, palestra, apresentação, entre outros. Dessa forma, foi possível adquirir os trabalhos que mais se alinhavam ao objetivo proposto no mapeamento sistemático.

Primeiramente, foi feita uma leitura e apreciação do título e resumo dos EP, originados do estágio anterior, e então aplicados os critérios de exclusão escolhidos. A seguir, é apresentado o quantitativo dos EP selecionados nessa etapa, por base de pesquisa e por ano de publicação (Tabela 3 ). 
Tabela 3: Quantidade de EP selecionados na etapa de Extração de Dados

\begin{tabular}{|c|c|c|c|c|}
\hline Base de Busca & Quantidade de EP & & Ano de Publicação & Quantidade de EP \\
\hline IEEE Xplore & 14 & 2014 & 03 \\
\hline Springer Link & 13 & 2015 & 17 \\
\hline ACM Digital Library & 02 & 2016 & 09 \\
\hline Scopus & 10 & 2017 & 06 \\
\hline Scielo & 01 & 2018 & 08 \\
\hline SAGE journals & 07 & 2019 & 05 \\
\hline Holos & 01 & $\mathbf{2}$ & \multirow{4}{*}{ Total } \\
\hline Total & $\mathbf{4 8}$ & & \\
\cline { 1 - 1 } & &
\end{tabular}

\subsection{Análise e Síntese}

Nesta etapa, foram determinados e aplicados critérios qualitativos (CQ) (Tabela 4) que nortearam o estudo qualitativo dos EP.

Tabela 4: Avaliação da Qualidade

\begin{tabular}{|l|}
\hline \multicolumn{1}{|c|}{ Critérios de Qualidade } \\
\hline - CQ1. EP relaciona tempo e clima em algum processo de ensino-aprendizagem? \\
- CQ2. EP apresenta de forma apropriada os métodos usados para coleta de dados? \\
- CQ3. EP responde à problemática proposta nesta pesquisa? \\
- CQ4. EP trata de utilização de tecnologias digitais em problemas de mudanças climáticas no \\
processo de ensino-aprendizagem? \\
- CQ5. EP apresenta abordagens pedagógicas relacionadas a mudanças climáticas de modo \\
interdisciplinar no processo de ensino-aprendizagem?
\end{tabular}

Para cada EP, iniciou-se a etapa de leitura e avaliação, através da qual se buscou descobrir quais realmente tinham relações com a questão de pesquisa, com base nos CQ. Para todo CQ, foi estabelecida a condição de Atende (AT) e Não Atende (NA). Também, foi aplicado um Índice de Aceitação (IA) do EP, onde zero para os que não atendiam a nenhuma AT, e cinco para os que atendiam a todos os CQ com condição AT. Os EP resultantes com IA igual ou maior a três foram classificados como Artigo s Aceitos (AA), o restante como Artigos Rejeitados (AR). Na Tabela 5, é exposta a identificação dos EP avaliados, com um identificador (ID), referência e situação de cada CQ. As referências completas dos EP estão disponíveis no link: https://bit.ly/2N7k1mL.

Tabela 5: Avaliação qualitativa dos EP

\begin{tabular}{|c|l|c|c|c|c|c|c|c|}
\hline ID & \multicolumn{1}{|c|}{ Referência } & CQ1 & CQ2 & CQ3 & CQ4 & CQ5 & IA & Resultado \\
\hline 01 & {$[$ Angel et al. 2015] } & AT & AT & AT & AT & AT & 5 & AA \\
\hline 02 & {$[$ Teijón et al. 2018] } & AT & AT & AT & AT & AT & 5 & AA \\
\hline 03 & {$[$ Silva et al. 2016] } & AT & AT & AT & NA & AT & 4 & AA \\
\hline 04 & {$[$ Prasad e Mogla 2016] } & AT & AT & AT & NA & AT & 4 & AA \\
\hline 05 & {$[$ Wyrwa 2018] } & AT & AT & AT & NA & AT & 4 & AA \\
\hline 06 & {$[$ Santos et al. 2016] } & AT & AT & AT & AT & AT & 5 & AA \\
\hline 07 & {$[$ Baideme et al. 2014] } & NA & AT & NA & NA & AT & 2 & AR \\
\hline 08 & {$[$ Lascano et al. 2015] } & NA & AT & NA & NA & AT & 2 & AR \\
\hline 09 & {$[$ Yamazaki et al. 2014] } & NA & AT & NA & NA & AT & 2 & AR \\
\hline 10 & {$[$ Typhina 2015] } & NA & AT & NA & AT & NA & 2 & AR \\
\hline 11 & {$[$ Christensen e Knezek 2016] } & AT & AT & NA & NA & AT & 3 & AA \\
\hline 12 & {$[$ Aziz et al. 2018] } & NA & AT & NA & NA & AT & 2 & AR \\
\hline 13 & {$[$ Gomes et al. 2016] } & AT & NA & NA & NA & 1 & AR \\
\hline 14 & {$[$ Cruz 2015] } & NA & NA & NA & NA & NA & 0 & AR \\
\hline 15 & {$[$ Chan 2015] } & AT & NA & AT & NA & AT & 3 & AA \\
\hline 16 & {$[$ Abdullah 2016] } & NA & NA & AT & NA & AT & 3 & AA \\
\hline 17 & {$[$ Ferreira et al. 2016] } & NA & AT & NA & 2 & AR \\
\hline
\end{tabular}




\begin{tabular}{|c|c|c|c|c|c|c|c|c|}
\hline 18 & [Boakye 2015] & AT & AT & AT & NA & NA & 3 & $\mathrm{AA}$ \\
\hline 19 & [Bieler et al. 2017] & AT & AT & AT & NA & NA & 3 & $\mathrm{AA}$ \\
\hline 20 & [Han 2015] & AT & NA & $\mathrm{NA}$ & NA & NA & 1 & AR \\
\hline 21 & [Sung 2015] & NA & $\mathrm{NA}$ & $\mathrm{NA}$ & NA & $\mathrm{NA}$ & 0 & $\mathrm{AR}$ \\
\hline 22 & [Læssøe e Mochizuki 2015] & NA & AT & NA & NA & $\mathrm{NA}$ & 1 & AR \\
\hline 23 & [Mochizuki e Bryan 2015] & AT & AT & AT & NA & AT & 4 & $\mathrm{AA}$ \\
\hline 24 & [Trajber e Mochizuki 2015] & AT & AT & AT & NA & AT & 4 & $\mathrm{AA}$ \\
\hline 25 & [Moço et al. 2016] & AT & AT & AT & AT & AT & 5 & $\mathrm{AA}$ \\
\hline 26 & [Jovanovic et al. 2016] & AT & AT & AT & NA & AT & 4 & $\mathrm{AA}$ \\
\hline 27 & [Lusk et al. 2017] & NA & AT & AT & NA & NA & 2 & AR \\
\hline 28 & [Sarıkaya e Saraç 2018] & AT & AT & NA & NA & NA & 2 & AR \\
\hline 29 & [Schmitt-Harsh e Harsh 2017] & NA & AT & NA & NA & NA & 1 & AR \\
\hline 30 & [Gold et al. 2015] & AT & AT & AT & NA & AT & 4 & $\mathrm{AA}$ \\
\hline 31 & [Foss e Ko 2019] & AT & AT & NA & NA & NA & 2 & AR \\
\hline 32 & [Clark e Zeegers 2015] & AT & AT & AT & NA & AT & 4 & $\mathrm{AA}$ \\
\hline 33 & [Tabuenca et al. 2019] & AT & AT & AT & AT & AT & 5 & $\mathrm{AA}$ \\
\hline 34 & [Camasso e Jagannathan 2018] & AT & AT & AT & NA & AT & 4 & $\mathrm{AA}$ \\
\hline 35 & [Morrison 2018] & AT & AT & AT & NA & AT & 4 & $\mathrm{AA}$ \\
\hline 36 & [Landrum et al. 2017] & NA & AT & NA & NA & $\mathrm{NA}$ & 1 & AR \\
\hline 37 & [Carey e Gougis 2017] & AT & AT & AT & AT & AT & 5 & $\mathrm{AA}$ \\
\hline 38 & [Rojas et al. 2015] & AT & AT & AT & NA & AT & 4 & $\mathrm{AA}$ \\
\hline 39 & [Cole 2019] & NA & AT & NA & NA & NA & 1 & AR \\
\hline 40 & [Bodzin e Fu 2014] & AT & AT & AT & AT & AT & 5 & $\mathrm{AA}$ \\
\hline 41 & [Visintainer e Linn 2015] & AT & AT & AT & AT & AT & 5 & $\mathrm{AA}$ \\
\hline 42 & [Mandrikas et al. 2017] & AT & AT & AT & NA & AT & 4 & $\mathrm{AA}$ \\
\hline 43 & [Lin et al. 2019] & NA & AT & $\mathrm{NA}$ & NA & $\mathrm{NA}$ & 1 & AR \\
\hline 44 & [Karahan e Roehrig 2015] & AT & AT & AT & AT & AT & 5 & $\mathrm{AA}$ \\
\hline 45 & [Fokides e Chachlaki 2019] & NA & AT & NA & NA & $\mathrm{NA}$ & 1 & AR \\
\hline 46 & [Puttick e Tucker-Raymond 2018] & AT & AT & AT & AT & AT & 5 & $\mathrm{AA}$ \\
\hline 47 & [Bush et al. 2018] & AT & AT & AT & AT & AT & 5 & $\mathrm{AA}$ \\
\hline 48 & [Belland et al. 2015] & NA & AT & $\mathrm{NA}$ & NA & NA & 1 & $\mathrm{AR}$ \\
\hline
\end{tabular}

\section{Resultados}

Após a análise qualitativa, 28 EP foram aceitos para o propósito do mapeamento sistemático. Na Tabela 6, é visto um panorama de EP que passaram pelas análises, assim como o percentual conforme EP aceitos e rejeitados.

Tabela 6. Panorama dos EP analisados

\begin{tabular}{|c|c|c|c|}
\hline Etapa & EP analisados & EP aceitos & EP rejeitados \\
\hline Extração de Dados & 192 & $48(25 \%)$ & $144(75 \%)$ \\
\hline Análise Qualitativa & 48 & $28(58,3 \%)$ & $20(41,7 \%)$ \\
\hline
\end{tabular}

$\mathrm{Na}$ Figura 1, é apresentado um Mapa Mental com a descrição referente às principais temáticas e aos seus respectivos autores na Análise Qualitativa. Um mapa mental é visto como uma técnica que ajuda em processos de organização do pensamento, ou seja, auxilia na hierarquização do pensamento e no melhor entendimento das informações sobre algum conteúdo [Buzzan 2005].

No Mapa Mental, as temáticas dos EP foram agrupadas em: Aplicação - estudos que unicamente expõem a necessidade de introdução do tema de mudanças climáticas nas escolas, sem a descrição de como inserir e avaliar, basicamente enfatiza a importância do tratamento das mudanças climáticas na educação; Jogo - apre sentação da temática com estratégias lúdicas para desenvolver uma atmosfera de cooperação e 
motivação para entendimento sobre as mudanças do clima, propiciando uma relação dinâmica e interativa no processo de aprendizagem; Capacitação - unidade de ensino com o objetivo de obter e/ou melhorar o conhecimento sobre mudanças climáticas; e Software educacional - programa de computador que tem por objetivo atender as necessidades e pretensões pedagógicas sobre a conscientização ambiental e entendimento das causas e consequências das mudanças do clima.

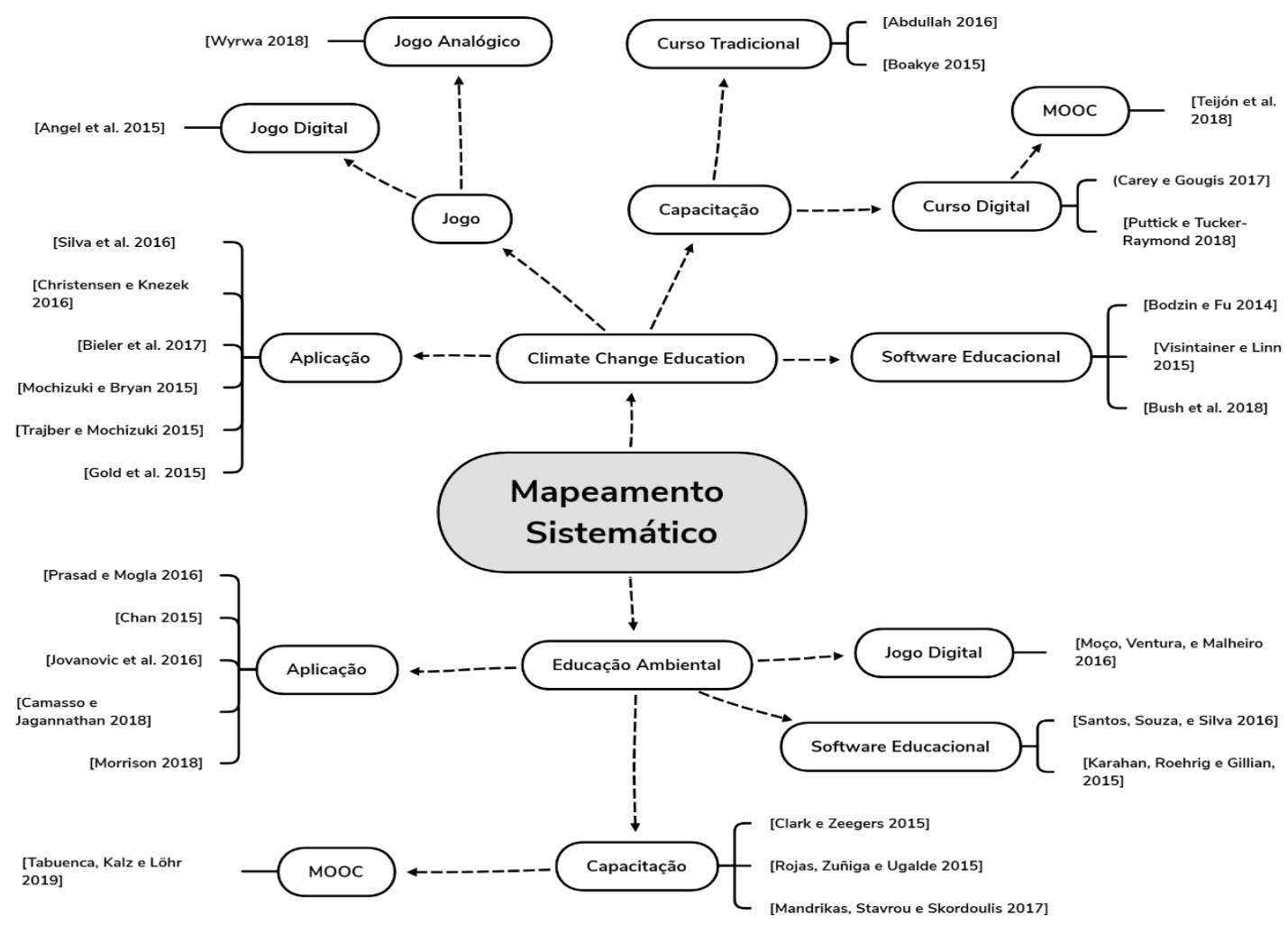

Figura 1. Mapa Mental com as temáticas e seus respectivos autores

Foram também analisadas as principais contribuições e temáticas abordadas nos EP que foram considerados aprovados. A Tabela 7 consolida-os com uma síntese.

Tabela 7. Tabela dos EP analisados pelos critérios qualitativos

\begin{tabular}{|c|l|}
\hline ID & \multicolumn{1}{c|}{ Síntese da Contribuição } \\
\hline 01 & $\begin{array}{l}\text { Discutido sobre os fundamentos teóricos de um jogo sério que visa facilitar o entendime nto e } \\
\text { a ação sobre as mudanças climáticas locais. Ojogo abrange arte, ciência e tecnologia para } \\
\text { comunicar uma visão sobre os desafios e soluções para o combate às mudanças climáticas. }\end{array}$ \\
\hline 02 & $\begin{array}{l}\text { Descreve um curso para explicar as causas das mudanças climáticas. O curso é um MOOC } \\
\text { (Massive Online Open Course) para professores do ensino fundamental e médio. Com o } \\
\text { conhecimento adquirido, é possível contribuir na preparação das jovens gerações para } \\
\text { mobilizar a sociedade diante do problema de mudanças climáticas. }\end{array}$ \\
\hline 03 & $\begin{array}{l}\text { Busca analisar como a interdisciplinaridade na educação, utilizando a CCE, podem facilitar a } \\
\text { construção de processos de mitigação e adaptação para as mudanças climáticas. }\end{array}$ \\
\hline 04 & $\begin{array}{l}\text { Utiliza EA como abordagem, sugerindo a formulação de uma política educacional inovadora, } \\
\text { com o objetivo de desenvolver conscientização. Sugere formas multidisciplinares de ensino } \\
\text { através da educação ao ar livre e experimental. }\end{array}$ \\
\hline 05 & $\begin{array}{l}\text { Apresenta um jogo para familiarizar alunos com estratégias para diminuir os efeitos das } \\
\text { mudanças climáticas. A principal ferramenta usada no jogo é um formulário de papel, que } \\
\text { constitui um scaffolding que fornece o material de aprendizagem de suporte. }\end{array}$ \\
\hline
\end{tabular}

" "Scaffolding" (andaimes) visa designar a ções sustentadas por a dultos em que cria nças podem expandir as suas habilidades e conhecimentos a pontos mais elevados de capacidade e sa ber [Va sconcelos 1999]. 


\begin{tabular}{|c|c|}
\hline 06 & $\begin{array}{l}\text { Proposta de aplicativo voltado à educação e a preservação ambiental em comunidades } \\
\text { urbanas. Visa promover os objetivos da EA, conforme a Declaração de Tbilisi [Unesco 1978]. }\end{array}$ \\
\hline 11 & $\begin{array}{l}\text { Demonstra mudanças anteriores e posteriores à aplicação de atividades práticas sobre a } \\
\text { energia e clima. Executado no ensino médio de } 04 \text { escolas de } 03 \text { Estados dos EUA, } \\
\text { orientando a solução de problemas reais sobre mudanças climáticas e nas intenções dos } \\
\text { alunos de agir para diminuir o ritmo das mudanças climáticas. }\end{array}$ \\
\hline 15 & $\begin{array}{l}\text { Utilizando aprendizado ativo com alunos trabalhando em grupos para coletar informações, } \\
\text { fazer uma análise e identificar os vínculos entre o padrão de intensidade das chuvas ao longo } \\
\text { dos anos com deslizam entos de terra localizados e inundações. }\end{array}$ \\
\hline 16 & $\begin{array}{l}\text { Abordagem no ensino-aprendizado de um curso de graduação superior, com conteúdo } \\
\text { multidisciplinar com atualidades sobre questões verdes, mudanças climáticas e sociais. }\end{array}$ \\
\hline 18 & $\begin{array}{l}\text { Sugere alterações nos currículos de ensino em Gana, rela cionando o conhecimento adquirido } \\
\text { sobre a educação em mudanças climáticas. Dessa forma, sendo possível a prom oção de uma } \\
\text { educação eficaz sobre as mudanças climáticas no país. }\end{array}$ \\
\hline 19 & $\begin{array}{l}\text { Analisa o envolvimento com o planejamento e aplicação de CCE do Canadá. Compara } 13 \\
\text { planos de ação climática e } 90 \text { documentos educacionais. Sugere ajustes no en sino do país } \\
\text { para se adequar às tendências internacionais de CCE. }\end{array}$ \\
\hline 23 & $\begin{array}{l}\text { Destaca o papel crítico que a educação pode e deve desempenhar para tratar sobre o tema e } \\
\text { como obter respostas às mudanças climáticas em toda a sua complexidade. Fornece } \\
\text { justificativas para o motivo pelo qual a CCE deve ser abordada. }\end{array}$ \\
\hline 24 & $\begin{array}{l}\text { Mapeia e explica políticas, estratégias e iniciativas do Brasil relacionadas à CCE, no contexto } \\
\text { geral de EA e EDS. }\end{array}$ \\
\hline 25 & $\begin{array}{l}\text { Aborda a possibilidade de um jogo pedagógico guiar o aluno/jovem/cidadão a pensar, a } \\
\text { levantar hipóteses e a consolidar o seu conhecimento conscientizando-se de que to dos são } \\
\text { parte do problema envolvendo mudanças climáticas e, em simultâneo, possíveis soluções. }\end{array}$ \\
\hline 26 & $\begin{array}{l}\text { Analisa tendências relacionadas a EA no ensino de geografia e determina a correlação entre } \\
\text { os elementos do processo de ensino, tais como: presença de tópicos ambientais no ensino de } \\
\text { geografia, avaliação do conhecimento dos alunos e atitudes dos professores sobre a } \\
\text { importância do tema de mudanças climáticas. }\end{array}$ \\
\hline 30 & $\begin{array}{l}\text { Relata a elaboração e os resultados do programa de aprendizado ativo de videografia da Lens } \\
\text { on Climate Change que visava aumentar a conscientização e a compreensão dos alunos } \\
\text { sobre como as mudanças climáticas afetam suas vidas. }\end{array}$ \\
\hline 32 & $\begin{array}{l}\text { Proposta de um curso focado em aumentar a conscientização dos alunos de pós-graduação } \\
\text { sobre sustentabilidade ambiental, de uma perspectiva de pensamento sistêmico com a } \\
\text { Aprendizagem Baseada em Problemas (ABP). }\end{array}$ \\
\hline 33 & $\begin{array}{l}\text { Implementa um MOOC como uma ferramenta massiva, focada na ação, para explorar e } \\
\text { disseminar maneiras de enfrentar o problema global do lixo marinho de uma perspectiva } \\
\text { educacional, através de um aprendizado orientado à ação. }\end{array}$ \\
\hline 34 & $\begin{array}{l}\text { Apresenta resultados do programa Nurture thru Nature (NtN), uma ação em escolas primárias } \\
\text { nas disciplinas de ciências naturais e EA, projetada para ajudar crianças socialmente } \\
\text { vulneráveis a aumentar o conhecimento sobre preservação ambiental e fortalecer o } \\
\text { desempenho acadêmico geral. }\end{array}$ \\
\hline 35 & $\begin{array}{l}\text { Relata as descobertas de um estudo qualitativo de conversas em grupo sobre EA com } \\
\text { professores. Assim, foi visto que os participantes compartilharam seus conhecimentos } \\
\text { informando os alunos sobre questões ambientais e políticas sociais, mostrando que a EA não } \\
\text { é uma área de conteúdo especializada e singular, mas um campo interdisciplinar, integral às } \\
\text { experiências cotidianas de aprendizagem. }\end{array}$ \\
\hline 37 & $\begin{array}{l}\text { Examina ganhos de aprendizagem resultantes de um curso (Lake Modeling da EDDIE) para } \\
\text { estudantes de biologia e estudantes de ciências, utilizando ferramentas de modelagem. Os } \\
\text { resultados sugerem que a modelagem é uma ferramenta poderosa para estimular o } \\
\text { aprendizado dos alunos sobre os efeitos das mudanças climáticas. }\end{array}$ \\
\hline 38 & $\begin{array}{l}\text { Propõe um programa educacional global para os níveis de ensino fundamental e médio. } \\
\text { Objetiva desenvolver o pensamento científico e o interesse pela ciência em estudantes } \\
\text { através da pesquisa em hidrologia permitindo relacionar a ciência com questões ambientais. }\end{array}$ \\
\hline 40 & $\begin{array}{l}\text { Curso sobre mudanças climáticas para estudantes de ensino fundamental, integrando a } \\
\text { tecnologia geoespacial Google Earth e outras atividades de aprendizado baseadas na Web } \\
\text { para apoiar o entendimento dos alunos. }\end{array}$ \\
\hline 41 & $\begin{array}{l}\text { Relata o impacto de tecnologia para melhorar a compreensão das mudanças climáticas. } \\
\text { Implementa um ambiente de investigação na Web (WISE), usando modelos NetLogo } \\
\text { (ambiente de modelagem programável para simular fenômenos naturais e sociais) com }\end{array}$ \\
\hline
\end{tabular}




\begin{tabular}{|c|l|}
\hline 42 & $\begin{array}{l}\text { crianças do ensino fundamental. Os resultados mostram que a compreensão do efeito estufa } \\
\text { pelos alunos oferece uma base para a construção de conexões entre o uso diário de en ergia } \\
\text { e o aumento da temperatura global. }\end{array}$ \\
\hline $\begin{array}{l}\text { Curso com contexto científico autêntico, ou seja, dados meteorológicos atualizados } \\
\text { diariamente sobre problemas ambientais com fenômenos meteorológicos, para prof essores } \\
\text { do ensino fundamental. Composto por uma sequência de ensino-aprendizagem sobre } \\
\text { poluição do ar, a contribuição do vento e da topog rafia na concentração de poluentes do ar. }\end{array}$ \\
\hline 44 & $\begin{array}{l}\text { Construção de artefatos multimídiapor alunos, com redes sociais (rede social Ning) para fins } \\
\text { de EA. O estudo indicou o aprimoramento da consciência ambiental dos alunos e a } \\
\text { necessidade percebida de ativismo foi elevada em diferentes escalas. }\end{array}$ \\
\hline 46 & $\begin{array}{l}\text { Curso para meninas do ensino médio, focado no uso do ambiente de programação visual } \\
\text { Scratch, para projetar jogos para ensinar outras pessoas sobre mudanças climáticas. }\end{array}$ \\
\hline 47 & $\begin{array}{l}\text { Comparou o uso educacional de um modelo climático global (em inglês, global climate model- } \\
\text { GCM) da National Aeronautics and Space Administration (NASA) com outras tecnologias de } \\
\text { educação climática desenvolvidas para uso em sala de aula que incluíam interfaces e } \\
\text { processos mais simples. Assim, foi visto como essas tecnologias de educação climática } \\
\text { afetam a evolução do entendimento de alunos sobre Mudanças Climáticas Antropogênicas } \\
\text { Globais (MCAG), através de aprendizagem baseada em modelos. }\end{array}$ \\
\hline
\end{tabular}

\section{Discussões}

A partir dos resultados obtidos no mapeamento sistemático da literatura, foi possível identificar quais as estratégias educacionais mais utilizadas para a compreensão das causas e impactos das mudanças climáticas. As abordagens pedagógicas Climate Change Education (CCE) e Educação Ambiental (EA) foram as mais citadas e utilizadas nos estudos analisados. A CCE é uma metodologia que objetiva melhorar o entendimento da dinâmica entre as ações antrópicas e as mudanças do clima, proporcionando a conscientização sobre a importância da realização dos meios de mitigar e adaptar os seus efeitos [Læssøe et al. 2009; Bangay e Blun 2010]. A EA é uma forma interdisciplinar e dinâmica de educação, com foco no meio ambiente e nos sistemas ecológicos, abrangendo o estudo de mudanças nos sistemas terrestres e ambientais no decorrer do tempo [Prasad e Mogla 2016].

$\mathrm{Na}$ observação dos resultados do mapeamento sistemático, como resposta à questão de pesquisa formulada, temos que: $16 \mathrm{EP}$ tratam sobre CCE e $12 \mathrm{EP}$ tratam de EA. Com relação aos EP de CCE: 5 EP tratam de capacitações como habilitadores do aprendizado da temática, 3 EP relacionam softwares educacionais à abordagem, 2 EP expõem jogos (analógicos ou digitais) em seus relatos, e 6 EP tratam de questões sobre os efeitos do uso de CCE na escola. Com relação aos EP de EA: 4 EP versam sobre capacitações (cursos) sobre o tema, 2 EP relacionam softwares educacionais ao conteúdo, 1 EP envolve jogos (analógicos ou digitais) sobre a temática e 5 EP colocam questões sobre os efeitos da aplicação de EA no meio escolar.

A reduzida quantidade de EP que relacionam mudanças climáticas a softwares educacionais pode ser vista como uma oportunidade para desenvolvimento de pesquisas que resultem em softwares específicos para introdução das mudanças climáticas nas múltiplas disciplinas escolares. Uma das propensões que mais se evidenciaram entre as pesquisas identificadas é a utilização de capacitações (cursos) tratando da temática, elas podem ser consideradas como uma forma de aliar elementos da abordagem fora da sala de aula possibilitando associar conhecimentos essenciais para obter uma percepção integrada, ajudando os alunos a desenvolver um entendimento dos principais mecanismos associados às mudanças do clima. Há várias propostas que fomentam o uso de estratégias educacionais mostrando diagnósticos, políticas e projetos no ensino- 
aprendizado em mudanças climáticas. Finalmente, a reduzida quantidade de jogos digitais como ferramentas para auxiliar o processo de ensino e aprendizagem envolvendo as mudanças climáticas configura-se outra oportunidade de investigação.

Observou-se que mesmo havendo publicações envolvendo diversos tipos de tecnologias digitais, não foi encontrado estudo sobre a transformação digital na educação tendo como entrada o tratamento das mudanças climáticas. A Transformação Digital pode ser considerada um processo de aculturação pelo qual indivíduos, times e organizações são levados a mudar paulatinamente de comportamentos e estruturas analógicos para plataformas digitais [Meira e Neves 2019]. Portanto, observa-se que existe uma área promissora a ser explorada, na forma de abordagem pedagógica, ocorrendo através da adaptação de professores e alunos na aplicação de tecnologias digitais no cotidiano das atividades escolares, pela utilização de tecnologias digitais no ambiente social ou pela adequação de tecnologias acessíveis do mercado de trabalho. A transformação digital possui a capacidade de alterar o ambiente em que ela for colocada em prática, alterando até mesmo o cotidiano das pessoas envolvidas.

\section{Conclusão}

Neste artigo, foram apresentados e discutidos a sistemática e os resultados de um mapeamento da literatura, conduzido com o objetivo de identificar as práticas, ferramentas e tecnologias digitais usadas nas abordagens educacionais direcionadas a introduzir a temática de mudanças climáticas no processo de ensino-aprendizagem. As abordagens Climate Change Education (CCE) e Educação Ambiental (EA) foram as que fundamentaram a maior parte dos estudos analisados e são geralmente utilizados os seguintes meios práticos no processo de ensino-aprendizagem: capacitações específicas (cursos), jogos (analógicos e digitais), softwares educacionais e exposição do tema.

Apesar de vários estudos relatarem o uso de tecnologia digital como habilita dor para introdução das mudanças climáticas na educação, não foi encontrado qualquer relato sobre a aplicação do paradigma de transformação digital na edu cação para es se propósito. Portanto, constitui-se uma oportunidade de pesquisa o desenvolvimento de uma abordagem de transformação digital aplicada ao processo de ensino-aprendizagem para tratar a multidisciplinaridade das mudanças climáticas, considerando os conceitos, princípios e boas práticas das abordagens educacionais como CCE e EA e o uso das tecnologias digitais, visando incorporar nos estudantes a mudança comportamen tal por meio da conscientização e compreensão das causas e impactos das mudanças climáticas.

\section{Referências}

Bangay, C. and Blum, N. (2010). "Education responses to climate change and quality: Two parts of the same agenda?". International Journal of Educational Development, 30(4), 359-368.

Buzzan, T. (2005). "Mapas Mentais e sua elaboração: um sistema definitivo de pensamento que transforma a sua vida". São Paulo: Cultrix.

Cherry, L. (2011). Young voices on climate change: The Paul F-Brandwein 2010 NSTA lecture. Journal of Science Education and Technology, 20(2), 208-213.

IPCC. (2007). Climate Change 2007: Synthesis Report. Contribution of Working Groups I, II and III to the Fourth Assessment Report of the Intergovernmental Panel on Climate Change. IPCC, Geneva, Switzerland, 104 pp. 
IPCC (2019). Summary for Policymakers. In: Climate Change and Land: an IPCC special report on climate change, desertification, land degradation, sustainable land management, food security, and greenhouse gas fluxes in terrestrial. IPCC, Geneva, Switzerland.

Læssøe, J., Schnack, K., Breiting, S., Rolls, S., Feinstein, N. and Goh, K. C. (2009). "Climate change and sustainable development: The response from education. A crossnational report from international alliance of leading education institutes". The Danish School of Education, Aarhus University.

Magalhães, C. V., Santos, R. E., Silva, F. Q. and Gomes, A. S. (2013). Caracterizando a pesquisa em informática na educação no Brasil: um mapeamento sistemático das publicações do SBIE. In Brazilian Symposium on Computers in Education (Simpósio Brasileiro de Informática na Educação-SBIE) (Vol. 24, No. 1, p. 22).

Meira, S. and Neves, A. (2019) "Strateegia - framework de transformação digital". The Digital Strategy Company, https://www.tds.com.pe/framework/, Fevereiro.

Mochizuki, Y. and Bryan, A. (2015). Climate change education in the context of education for sustainable development: Rationale and principles. Journal of Education for Sustainable Development, 9(1), 4-26.

ONU. (2020). "Objetivo 13. Tomar medidas urgentes para combater a mudança climática e seus impactos". https://nacoesunidas.org/pos2015/ods13/, Fevereiro.

Petersen, K.; Feldt, R.; Mujtaba, S.; Mattsson, M. (2008) Systematic Mapping Studies in Software Engineering. 12th International Conference on Evaluation and Assessment in Software Engineering (EASE). University of Bari, Italy.

Pinho, R. M. L. and Granziera, M. L. M. (2019). "Efeitos das Mudanças Climáticas na Zona Costeira: O Caso de Santos". Leopoldianum, 45(125), 12.

Prasad, A. and Mogla, R. (2016). Environmental education: Component of sustainable development. In 2016 IEEE Region 10 Humanitarian Technology Conference (R10HTC) (pp. 1-4). IEEE.

Puttick, G. and Tucker-Raymond, E. (2018). Building systems from scratch: An exploratory study of students learning about climate change. Journal of Science Education and Technology, 27(4), 306-321.

Reboita, M. S., Krusche, N., Ambrizzi, T. and Rocha, R. P. D. (2012). Entendendo o Tempo e o Clima na América do Sul. RI FURG.

Sharma, A. (2012). Global climate change: What has science education got to do with it? Science \& Education, 21(1), 33-53.

UNESCO. (1978). Tblisi Conference Recommendations. Connect, 3(1).

Vasconcelos, T. (1999). "Encontrar as formas de ajuda necessária: O conceito de" scaffolding" (pôr, colocar andaimes), a sua aplicação em educação pré-escolar". Revista Inovação, 12(2), 7-24.

Visintainer, T. and Linn, M. (2015). Sixth-grade students' progress in understanding the mechanisms of global climate change. Journal of Science Education and Technology, 24(2-3), 287-310. 